\title{
Large time asymptotics of the doubly nonlinear equation in the non-displacement convexity regime
}

\author{
Martial Agueh , Adrien Blanchet ${ }^{\dagger}$ \& José A. Carrillo
}

June 10th, 2009

\begin{abstract}
We study the long-time asymptotics of the doubly nonlinear diffusion equation $\rho_{t}=$ $\operatorname{div}\left(\left|\nabla \rho^{m}\right|^{p-2} \nabla\left(\rho^{m}\right)\right)$ in $\mathbb{R}^{n}$, in the range $\frac{n-p}{n(p-1)}<m<\frac{n-p+1}{n(p-1)}$ and $1<p<\infty$ where the mass of the solution is conserved, but the associated energy functional is not displacement convex. Using a linearisation of the equation, we prove an $L^{1}$-algebraic decay of the non-negative solution to a Barenblatt-type solution, and we estimate its rate of convergence. We then derive the nonlinear stability of the solution by means of some comparison method between the nonlinear equation and its linearisation. Our results cover the exponent interval $\frac{2 n}{n+1}<p<\frac{2 n+1}{n+1}$ where a rate of convergence towards self-similarity was still unknown for the $p$-Laplacian equation.
\end{abstract}

\section{Introduction}

In this work, we consider the doubly nonlinear equation defined for any $(t, x) \in(0, \infty) \times \mathbb{R}^{n}$ by

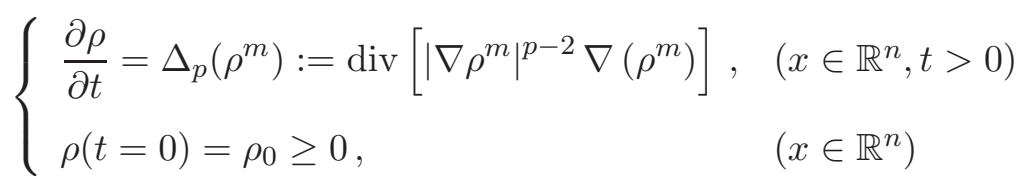

with $1<p<\infty, 0<m$ and $n \geq 3$. This class of equations contains the linear diffusion equation, $(p=2, m=1)$, commonly known as the heat equation, $\partial_{t} \rho=\Delta \rho$; the nonlinear diffusion equation $\partial_{t} \rho=\Delta \rho^{m}$, known as the porous medium equation $(p=2, m>1)$, or the fast diffusion equation $(p=2, m<1)$, and the gradient-dependent diffusion equation, $\partial_{t} \rho=\operatorname{div}\left(|\nabla \rho|^{p-2} \nabla \rho\right):=\Delta_{p} \rho$, that is, the $p$-Laplacian equation, $(p \neq 2, m=1)$. When $p \neq 2$ and $m \neq 1$, Eq. (1.1) is called the doubly nonlinear diffusion equation, due to the fact that its diffusion term depends non-linearly on both the unknown density $\rho$, and its gradient $\nabla \rho$. Such gradient-dependent diffusion equations appear in several models in non-Newtonian fluids [23], in

\footnotetext{
${ }^{*}$ Department of Mathematics and Statistics University of Victoria PO Box 3060 STN CSC Victoria, BC, Canada, V8W 3R4. E-mail: agueh@math.uvic.ca, Internet: http://www.math.uvic.ca/ agueh/

${ }^{\dagger}$ GREMAQ, Université de Toulouse, Manufacture des tabacs, 21 allée de Brienne, F-31000 Toulouse. E-mail: Adrien.Blanchet@univ-tlse1.fr, Internet: http://math.univ-tlse1.fr/blanchet/

${ }^{\ddagger}$ ICREA (Institució Catalana de Recerca i Estudis Avançats) and Departament de Matemàtiques, Universitat Autònoma de Barcelona, E-08193 Bellaterra, SPAIN. E-mail: carrillo@mat.uab.es, Internet: http://kinetic.mat.uab.es/ carrillo/
} 
glaciology [20, 8], and in turbulent flows in porous media [25]. For more details on these models, we refer to the recent monograph of Vázquez [33], and the references therein.

Assuming that the initial data is integrable, $\rho_{0} \in L^{1}\left(\mathbb{R}^{n}\right)$, it is known that (1.1) has a unique solution $\rho \in C\left([0, \infty), L^{1}\left(\mathbb{R}^{n}\right)\right)$, with $\rho(t) \in C^{1, \alpha}\left(\mathbb{R}^{n}\right)$ for some $\alpha \in(0,1)$, see for instance $[13,14,15,26]$. Moreover, starting with a non-negative initial data, $\rho_{0} \geq 0$, it is known that the solution $\rho(t)$ remains non-negative at all times. Furthermore for $n \geq 3$, there exists a critical exponent,

$$
m_{c}(p):=\frac{n-p}{n(p-1)},
$$

such that if $m>m_{c}(p)$, then the mass of the solution is conserved, $\int_{\mathbb{R}^{n}} \rho(t) \mathrm{d} x=\int_{\mathbb{R}^{n}} \rho_{0} \mathrm{~d} x$, while if $m<m_{c}(p)$, the solution vanishes in finite time, see [13,33] and the references therein. In particular, for the $p$-Laplacian equation, this corresponds to the critical $p$-exponent,

$$
p_{c}:=\frac{2 n}{n+1},
$$

above which the mass of the solution is conserved, while the solution disappears in finite time if $p<p_{c}$. Therefore, up to renormalising the mass of $\rho_{0}$ to unity, we can assume without loss of generality that, under the condition $m>m_{c}(p)$, the solution $\rho(t)$ of $(1.1)$ is a density in $\mathbb{R}^{n}$, for all times $t \geq 0$.

By similarity and scaling, it can be shown that, above the critical exponent $m_{c}(p)$, Eq.(1.1) has a unique self-similar solution $\rho_{D_{*}}$, whose initial value is the Dirac mass at the origin, that is, the fundamental solution of Eq.(1.1). In fact, among all the radially symmetric solutions of (1.1), this solution is the most concentrated whose initial data have the same mass as $\rho_{0}$. It is called the Barenblatt solution [4], and it is precisely:

$$
\rho_{D_{*}}(t, x)=\frac{1}{\left(\delta_{p} t\right)^{n / \delta_{p}}} u_{D_{*}}\left(\frac{x}{\left(\delta_{p} t\right)^{1 / \delta_{p}}}\right)
$$

where

$$
\delta_{p}:=n(p-1)\left(m-m_{c}(p)\right)>0,
$$

and

$$
u_{D_{*}}(y)= \begin{cases}\frac{1}{\sigma} \exp \left(-\frac{|p-1|^{2}}{p}|y|^{p /(p-1)}\right) & \text { if } \quad m=\frac{1}{p-1} \\ \left(D_{*}-\frac{m(p-1)-1}{m p}|y|^{p /(p-1)}\right)_{+}^{\frac{p-1}{m(p-1)-1}} & \text { if } \quad m \neq \frac{1}{p-1},\end{cases}
$$

with $\sigma$ and $D_{*}$ are uniquely determined by the mass conservation: $\left\|u_{D_{*}}\right\|_{L^{1}\left(\mathbb{R}^{n}\right)}=\left\|\rho_{D_{*}}(t)\right\|_{L^{1}\left(\mathbb{R}^{n}\right)}=$ $\left\|\rho_{0}\right\|_{L^{1}\left(\mathbb{R}^{n}\right)}$.

When $p=2$ and $m>1-2 / n$, the existence and uniqueness of the Barenblatt solution was proved by Friedmann and Kamin in [19]. Moreover, they showed that the solution $\rho(t)$ of the Cauchy problem converges to $\rho_{D_{*}}(t)$ w.r.t. the $L^{1}\left(\mathbb{R}^{n}\right)$-norm, as $t \rightarrow \infty$, with no rates. Rates of convergence were computed by Carrillo and Toscani [9] if $m>1$, independently by Del Pino and Dolbeault [16], and Otto [31] if $m \geq 1-1 / n$. The rates found in this range were generically optimal. In the range $1-2 / n<m<1-1 / n$, there were studies of the linearised problem by Carrillo, Lederman, Markowich and Toscani [10], and Denzler and McCann [12]. These linearisations were useful to obtain rates of decay for the nonlinear fast diffusion equation 
by Carrillo and Vázquez [11] and later by McCann and Slepčev [29], and Kim and McCann [21]. The decay rates obtained by using the linearisations are in general non optimal and is optimal in some sub-range, see [21].

When $p \neq 2$ and $m=1$, Kamin and Vázquez [22] proved existence and uniqueness of the Barenblatt solution $\rho_{D_{*}}$ for the $p$-Laplacian equation when $p>p_{c}$, along with an $L^{1}$-convergence of the solution $\rho(t)$ of the Cauchy problem to $\rho_{D_{*}}(t)$, with no rates. Their proof extends to the doubly nonlinear equation as long as $m>m_{c}(p)$, see [33]. Rates of convergence were computed by Del Pino and Dolbeault [17] when $p_{c}+1 /(n+1) \leq p<n$ for the $p$-Laplacian equation, but their rates are not optimal; see also a similar result for the doubly nonlinear equation in [18]. In [1,3], Agueh generalises previous results by deriving optimal rates for the convergence of the solution of the Cauchy problem (1.1) to $\rho_{D_{*}}(t)$, for all $m \geq m_{c}(p)+1 /(n(p-1))=$ $(n-p+1) /(n(p-1))$ and $p>1$. For instance, when $p=2$, this condition coincides with the case $m \geq 1-1 / n$, while for the $p$-Laplacian equation $(p \neq 2, m=1)$, it corresponds to $p \geq p_{c}+1 /(n+1)=(2 n+1) /(n+1)$, and therefore covers the range $p \geq n$ left in [17], but not the remaining exponent interval $2 n /(n+1)<p<(2 n+1) /(n+1)$. Similarly, for the doubly nonlinear diffusion equation, the rate of convergence remains unknown in the range

$$
m_{c}(p)<m<m_{c}(p)+\frac{1}{n(p-1)}=\frac{n-p+1}{n(p-1)} .
$$

Indeed, the proof of [3] is based on optimal transportation inequalities, which follows from the displacement convexity [28] of the energy functional associated with $(1.1)$, that is, $H^{F}(\rho)=$ $\int_{\mathbb{R}^{n}} F[\rho] \mathrm{d} x$, where

$$
F(x)= \begin{cases}\frac{1}{p-1} x \ln x & \text { if } \quad m=\frac{1}{p-1} \\ \frac{m x^{\gamma}}{\gamma(\gamma-1)} & \text { if } \quad m \neq \frac{1}{p-1}\end{cases}
$$

and

$$
\gamma:=m+\frac{p-2}{p-1}
$$

This energy functional is displacement convex if and only if $\gamma \geq 1-\frac{1}{n}$, or equivalently $m \geq$ $(n-p+1) /(n(p-1))$. This explains why the method of [3] does not extend to the interval (1.3).

The goal of this work is then precisely to derive a rate of convergence w.r.t the $L^{1}\left(\mathbb{R}^{n}\right)$-norm, of the non-negative solution $\rho$ of the Cauchy problem (1.1), to the Barenblatt solution $\rho_{D_{*}}(t)$, as $t \rightarrow \infty$, provided that $m$ belongs to the remaining exponent interval (1.3), that is,

$$
\frac{n-p}{n(p-1)}<m<\frac{n-p+1}{n(p-1)} \Leftrightarrow 1-\frac{q}{n}<\gamma<1-\frac{1}{n} .
$$

For convenience we rewrite the Cauchy problem (1.1) as:

$$
\begin{cases}\frac{\partial \rho}{\partial t}=\operatorname{div}\left\{\rho \nabla c^{*}\left[\nabla\left(F^{\prime} \circ \rho\right)\right]\right\}, & \left(x \in \mathbb{R}^{n}, t>0\right) \\ \rho(t=0)=\rho_{0}, & \left(x \in \mathbb{R}^{n}\right),\end{cases}
$$


where $c^{*}(x)=|x|^{p} / p$ is the Legendre transform of the convex function

$$
c(x)=\frac{|x|^{q}}{q}, \quad \frac{1}{p}+\frac{1}{q}=1 .
$$

By rescaling in time and space $\rho$ as follows:

$$
\rho(t, x)=\frac{1}{R(t)^{n}} u(\tau, y),
$$

where

$$
\tau=\ln R(t), \quad y=\frac{x}{R(t)}, \quad R(t)=\left(1+\delta_{p} t\right)^{1 / \delta_{p}}, \quad \delta_{p}=(p-1)(n m+1)+1-n,
$$

it is easy to show that $\rho$ solves (1.6) if and only if $u$ solves the rescaled convection-diffusion equation

$$
\begin{cases}\frac{\partial u}{\partial \tau}=\operatorname{div}\left\{u \nabla c^{*}\left[\nabla\left(F^{\prime} \circ u\right)\right]+u y\right\} & \left(y \in \mathbb{R}^{n}, \tau>0\right) \\ u(\tau=0)=\rho_{0} & \left(y \in \mathbb{R}^{n}\right) .\end{cases}
$$

Moreover, by conservation of mass there exists a unique $D_{*}$ such that the Barenblatt profile $u_{D_{*}}$ is the equilibrium solution of (1.9). Remark that in the considered range of exponents (1.5), $m(p-1)-1<0$, and then

$$
F(x)=\frac{m x^{\gamma}}{\gamma(\gamma-1)}, \quad \gamma:=m+\frac{p-2}{p-1} .
$$

Therefore, the Barenblatt profile is simply given by

$$
u_{D_{*}}(y)=\left(D_{*}+\frac{1-\gamma}{m} c(y)\right)^{\frac{1}{\gamma-1}}
$$

In fact, $u_{D_{*}}$ is the unique density function of same mass as $u_{0}$ which satisfies on its support,

$$
\nabla\left(F^{\prime} \circ u_{D_{*}}\right)=-\nabla c .
$$

The main result of our paper is the following:

Theorem 1.1 (Rates of convergence) Let $m, p$ be in the range (1.5), and let $u_{0}$ be a density such that there exist positive constants $D_{0}>D_{1}$ for which

$$
u_{D_{0}}(x) \leq \rho_{0}(x)=u_{0}(x) \leq u_{D_{1}}(x) \quad \forall x \in \mathbb{R}^{n} .
$$

Consider $u$ a solution to (1.9) with initial data $u_{0}$, there exists a unique $D_{*}$ such that $u(\tau)$ converges to the Barenblatt profile $u_{D_{*}}$ in $L^{1}\left(\mathbb{R}^{n}\right)$. Moreover, there exist a time $\tau_{0}$ and two positive constants $\lambda$ and $M=M\left(m, n, p, u_{0}, \tau_{0}\right)$ such that, for any time $\tau>\tau_{0}$

$$
\left\|u(\tau)-u_{D_{*}}\right\|_{L^{1}\left(\mathbb{R}^{n}\right)} \leq M e^{-\frac{\lambda}{2} \tau} .
$$


As a consequence, for a time large enough the corresponding solution $\rho(t)$ of $(1.1)$ converges to the Barenblatt solution $\rho_{D_{*}}(t)$, algebraically fast in the $L^{1}$-norm, at the rate $\lambda /\left(2 \delta_{p}\right)$ : there exist a time $t_{0}$ and a constant $C=C\left(m, n, p, \rho_{0}, t_{0}\right)$ such that, for any time $t>t_{0}$

$$
\left\|\rho(t)-\rho_{D_{*}}(t)\right\|_{L^{1}\left(\mathbb{R}^{n}\right)} \leq C t^{-\lambda /\left(2 \delta_{p}\right)},
$$

where $\delta_{p}=(p-1)(n m+1)+1-n$.

The main tool is the following relative free energy with respect to the Barenblatt solution $u_{D_{*}}$ defined by

$$
\mathcal{E}\left[u \mid u_{D_{*}}\right]:=\int_{\mathbb{R}^{n}}\left[F \circ u(y)-F \circ u_{D_{*}}(y)-F^{\prime} \circ u_{D_{*}}(y)\left(u(x)-u_{D_{*}}(y)\right)\right] \mathrm{d} y
$$

for any given $u \in L_{+}^{1}\left(\mathbb{R}^{n}\right)$. Its derivative along the flow of (1.6) is formally given by

$$
-\frac{\mathrm{d}}{\mathrm{d} \tau} \mathcal{E}\left[u(\tau) \mid u_{D_{*}}\right]=\mathcal{I}\left[u(\tau) \mid u_{D_{*}}\right]
$$

where

$$
\mathcal{I}\left[u(\tau) \mid u_{D_{*}}\right]:=\int_{\mathbb{R}^{n}} u(\tau, y) \nabla\left(F^{\prime} \circ u(\tau, y)+c(y)\right) \cdot\left(\nabla c^{*} \circ \nabla F^{\prime} \circ u(\tau, y)+y\right) \mathrm{d} y .
$$

In this paper, we prove that the relative entropy decays exponentially fast in the form

$$
\mathcal{E}\left[u(\tau) \mid u_{D_{*}}\right] \leq \mathrm{e}^{-\beta \tau} \mathcal{E}\left[u_{0} \mid u_{D_{*}}\right],
$$

for some $\beta>0$. This is obtained in two steps. First, we linearise (1.9) at the equilibrium solution $u_{D_{*}}$ by using the linear perturbation $u(\tau)=u_{D_{*}}+\epsilon v(\tau)$, and we show that the linearised version of the relative energy converges to 0 exponentially fast, as in [10]. For that, we use the Hardy-Poincaré inequality recently established by Blanchet, Bonforte, Dolbeault, Grillo and Vázquez in [5]. Next, following the strategy in [6], we try to compare the relative energy and the dissipation of the relative energy -that is, the Fisher information- for both linearised and nonlinear equations, to deduce the exponential decay (1.16) for the nonlinear equation. The main differences with respect to [6] lie in the fact that a direct relation between the linearised and the nonlinear Fisher information is not clear due to the singular characters at the origin of the weights when $1<p<2$. Therefore, we are forced to use a sort of regularised linearised Fisher information instead. Moreover, the control of the additional terms appearing in the regularised entropy dissipation of the linearised problem and in the relation between the entropy dissipations is more involved in our case.

We note that, based on our computations (see Remark 3.4), the Bakry-Emery approach used in [10], which consists of differentiation twice the relative energy $\mathrm{E}[v(\tau)]$ to estimate the spectral gap at the eigenvalue 0 , does not yield a positive result for our equation when $1<p<2$, and thus, a similar procedure to [11] for the doubly nonlinear equations is not feasible. Moreover, the Hardy-Poincaré inequality used here to establish the linear stability is actually valid on a larger interval, $m_{*}(p)<m<m_{c}(p)+\frac{1}{n(p-1)}$, which includes our interval $m_{c}(p)<m<m_{c}(p)+\frac{1}{n(p-1)}$, as $m_{*}(p)<m_{c}(p)$, where $m_{*}(p):=\frac{n-2 q}{n-q}+\frac{2-p}{p-1}$. Therefore, our linearisation result extends naturally to the interval $m_{*}<m \leq m_{c}(p)$ where mass conservation for the nonlinear equation fails. In this range, one needs to carefully define the right class of initial data and a substitute of the 
Barenblatt solution, as done in [6] when $p=2$. Here, we will not follow this path and we will restrict ourselves to the case $m_{c}(p)<m<m_{c}(p)+\frac{1}{n(p-1)}$ where mass is conserved to concentrate in the main new difficulties.

The paper is organised as follows. In Section 2, we review and introduce the main estimates on the solutions needed in the rest of the work. In particular, we prove the convergence of the solution $u(\tau)$ of (1.9) to the equilibrium solution $u_{D_{*}}$ in $C^{1}\left(\mathbb{R}^{n}\right)$, as $\tau \rightarrow \infty$, with no rate. Then in Section 3, we analyse a suitable linearised problem for which we apply an entropy-entropy dissipation argument based on Hardy-Poincaré inequalities. Finally, Section 4 is devoted to establish the exponential decay of $u(\tau)$ to $u_{D_{*}}$ by the comparison between linear and nonlinear relative entropy dissipations.

\section{Convergence without rate}

Let us start by reviewing some well-known facts about the global unique weak solutions associated to (1.1) in the range of exponents considered. They conserve mass for all times, i.e.,

$$
\int_{\mathbb{R}^{n}} \rho(t, x) \mathrm{d} x=\int_{\mathbb{R}^{n}} \rho_{0}(x) \mathrm{d} x \quad \forall t \in(0, \infty) .
$$

From now on, $D_{*}$ is the unique positive real such that

$$
\int_{\mathbb{R}^{n}} \rho_{0}(x) \mathrm{d} x=\int_{\mathbb{R}^{n}} u_{D_{*}}(x) \mathrm{d} x .
$$

Moreover, solutions of the Cauchy problem to (1.1) enjoy a comparison principle and the $L^{1}$ contraction property. Due to the change of variables (1.7)-(1.8), these properties hold for the solution $u$ of the nonlinear Fokker-Planck equation (1.9). Since in the rest of this paper we will only work with the scaled solutions of the nonlinear Fokker-Planck equation (1.9), from now on we will use $t$ instead of $\tau$ and $x$ instead of $y$ for the time and position variables respectively. The quotient function

is solution to

$$
w(t, x):=\frac{u(t, x)}{u_{D_{*}}(x)}
$$

$$
\frac{\partial w(t, x)}{\partial t}=\frac{1}{u_{D_{*}}(x)} \operatorname{div}\left\{w(t, x) u_{D_{*}}(x)\left(\nabla c^{*} \circ \nabla\left[F^{\prime} \circ\left(w(t, .) u_{D_{*}}\right)\right](x)-\nabla c^{*} \circ \nabla\left[F^{\prime} \circ u_{D_{*}}\right](x)\right)\right\} .
$$

Define

$$
W_{0}:=\inf _{x \in \mathbb{R}^{n}} \frac{u_{D_{0}}(x)}{u_{D_{*}}(x)} \leq \sup _{x \in \mathbb{R}^{n}} \frac{u_{D_{1}}(x)}{u_{D_{*}}(x)}:=W_{1} .
$$

A straightforward calculation gives

$$
W_{0}=\left(\frac{D_{*}}{D_{0}}\right)^{\frac{1}{1-\gamma}} \leq 1 \leq\left(\frac{D_{*}}{D_{1}}\right)^{\frac{1}{1-\gamma}}:=W_{1}
$$

with strict inequalities unless $\rho_{0}=u_{D_{*}}$. In terms of $w_{0}=u_{0} / u_{D^{*}}$, the "sandwich" assumption on the initial data (H1) of Theorem 1.1 can be rewritten as follows: there exist positive constants $D_{0}>D_{1}$ such that

$$
0<W_{0} \leq \frac{u_{D_{0}}(x)}{u_{D_{*}}(x)} \leq w_{0}(x) \leq \frac{u_{D_{1}}(x)}{u_{D_{*}}(x)} \leq W_{1}<\infty \quad \forall x \in \mathbb{R}^{n}
$$


Remark 2.1 Let us point out that the condition (H1) or (H1') in the case of the fast diffusion equation $(p=2)$ and in the corresponding range, $1-2 / n<m<1-1 / n$, is not restrictive. In fact, as a consequence of the global Harnack principle proved in [7], the hypothesis (H1) is satisfied by $\rho(t)$ for any $t>0$ with an initial data $u_{0} \in L_{+}^{1}\left(\mathbb{R}^{n}\right)$. In the present case, a similar Harnack principle, not available in the literature, would restrict the study of the asymptotic rates to this particular set of initial data.

Let us first remind the standard regularity theory of degenerate parabolic equations $[24,27,13$, $14,15,26]$ :

Lemma 2.2 (Interior regularity estimates) Let $\Omega$ be a bounded set and $\left\{u_{n}\right\}_{n}$ be a sequence of solutions to (1.9). If there exists $t_{0}$ and $0<C_{1} \leq C_{2}$ such that for all $t \geq t_{0}$

$$
C_{1} \leq u_{n}(t) \leq C_{2}
$$

then there exists $C$ such that for all $\alpha \in(0,1)$ and $t \geq t_{0},\left\|u_{n}(t)\right\|_{\mathcal{C}^{1, \alpha}} \leq C$.

As a consequence we have

Lemma 2.3 (Uniform $\mathcal{C}^{1, \alpha}$-estimates) Consider a solution $u \in C\left([0, \infty) ; L^{1}\left(\mathbb{R}^{n}\right)\right.$ ) of $(1.9)$ with initial data $u_{0}$ satisfying $(\mathrm{H} 1)$. If $w=u / u_{D^{*}}$ then for any $\alpha \in(0,1)$ and $t_{0} \in(0, \infty)$,

$$
\sup _{t \geq t_{0}}\|w(t)\|_{\mathcal{C}^{1, \alpha}\left(\mathbb{R}^{n}\right)}<\infty .
$$

Moreover, there exists $C>0$ such that for any $x \in \mathbb{R}^{n} \backslash B_{1}$

$$
|\nabla w(t, x)| \leq C \frac{w(t, x)}{|x|} \quad \forall t>0 .
$$

As a consequence,

$$
|\nabla u| \sim_{|x| \rightarrow \infty}|x|^{q /(\gamma-1)+1} .
$$

Proof. Due to the comparison principle and the hypothesis (H1), the function $u(t)$ is sandwiched between the two Barenblatt profiles for all times, i.e.,

$$
u_{D_{0}} \leq u(t) \leq u_{D_{1}} \quad t \geq 0
$$

and thus is uniformly bounded in $B_{2}$, the Euclidean ball of radius 2, uniformly in $t \geq t_{0}>0$. Due to Lemma 2.2, $u(t) \in \mathcal{C}^{1, \alpha}\left(B_{1}\right)$, for any $0 \leq \alpha<1$ hold uniformly in $t \geq t_{0}>0$. Consider $w=u / u_{D_{*}}$, then $w$ is also bounded in $\mathcal{C}^{1, \alpha}\left(B_{1}\right)$, for any $0 \leq \alpha<1$ uniformly in $t \geq t_{0}>0$. To deal with large values of $x$ we introduce, the rescaled function

$$
u_{\lambda}(t, x):=\lambda^{p /(1-m)} u(t, \lambda x)
$$

which is also solution to (1.9) but the annulus $B_{2 \lambda} / B_{\lambda}$ gets mapped into the annulus $\Omega_{1}:=B_{2} / B_{1}$. Note that all derivatives of the rescaled Barenblatt $u_{D_{*} / \lambda}$ are uniformly bounded from above and below since

$$
D^{\beta} u_{D_{*} / \lambda} \rightarrow C D^{\beta}\left(|x|^{\frac{q(p-1)}{m(p-1)-1}}\right) \quad \text { uniformly in } \Omega_{1} \text { as } \lambda \rightarrow \infty
$$


for any multi-index $\beta \in \mathbb{N}^{d}$. As a consequence, we get that $u_{\lambda}(t)$ is also uniformly bounded from above and below in $\Omega_{1}$ uniformly in $\lambda \geq 1$ and $t \geq t_{0}>0$. Again using the regularity theory of the degenerate parabolic equation, we deduce that the $\mathcal{C}^{1, \alpha}$-norm of $u_{\lambda}(t)$ and thus of $w_{\lambda}(t)$ in $\Omega_{1}$ is also uniformly bounded for $t \geq t_{0}$ and $\lambda \geq 1$ by a constant $C_{1}$. Going back in the $\lambda$-scaling we find a constant independent of $\lambda>1$ such that

$$
\frac{|\nabla w(t, \lambda x)|}{w(t, \lambda x)} \leq \frac{C_{1}}{\lambda} \quad \text { in }\left(t_{0}, \infty\right)
$$

in $\Omega_{1}$, and thus, the $\mathcal{C}^{1}$-norm of $w(t)$ in $\mathbb{R}^{n} / B_{1}$ is uniformly bounded. Similar scaling argument applies to the Hölder semi-norms. As a consequence of $(2.5)$

$$
|\nabla w(t, \lambda x)| \leq C_{1} \frac{w(t, \lambda x)}{\lambda} \leq 2 C_{1} \frac{w(t, \lambda x)}{\lambda|x|} .
$$

We thus obtain (2.3) for any $y=\lambda x \in B_{2 \lambda} \backslash B_{\lambda}$, and any $\lambda>0$.

Note that $\nabla u=u_{D} \nabla w+w \nabla u_{D}$ and thus we have (2.4).

From this we can obtain the following result regarding the evolution of the relative entropy to the stationary state.

Proposition 2.4 (Entropy/entropy production) Let $u \in C\left([0, \infty) ; L^{1}\left(\mathbb{R}^{n}\right)\right.$ ) be a solution of (1.9) for an initial data satisfying (H1), and consider the free energy $\mathcal{E}$ defined by (1.15). Its derivative along the flow of (1.9) is:

$$
\frac{\mathrm{d}}{\mathrm{d} t} \mathcal{E}\left[u(t) \mid u_{D_{*}}\right]=-\mathcal{I}\left[u(t) \mid u_{D_{*}}\right]
$$

where

$$
\mathcal{I}\left[u(t) \mid u_{D_{*}}\right]:=\int_{\mathbb{R}^{n}} u(t)\left[\nabla\left(F^{\prime} \circ u(t)-F^{\prime} \circ u_{D_{*}}\right)\right] \cdot\left[\nabla c^{*}\left(\nabla\left(F^{\prime} \circ u(t)\right)\right)-\nabla c^{*}\left(\nabla\left(F^{\prime} \circ u_{D_{*}}\right)\right)\right] \mathrm{d} y
$$

is the relative Fisher information of $u(t)$ w.r.t. $u_{D_{*}}$. Moreover, $\mathcal{I}\left[u(t) \mid u_{D_{*}}\right]=0$ if and only if $u=u_{D_{*}}$.

Proof. By performing formally integration by parts, we get

$$
\begin{aligned}
\frac{\mathrm{d}}{\mathrm{d} t} \mathcal{E}\left[u(t) \mid u_{D_{*}}\right] & =\int_{\mathbb{R}^{n}}\left[F^{\prime} \circ u(t)-F^{\prime} \circ u_{D^{*}}\right] \operatorname{div}\left\{u(t) \nabla c^{*}\left[\nabla\left(F^{\prime} \circ u(t)\right)\right]+u(t) y\right\} \mathrm{d} y \\
& =-\int_{\mathbb{R}^{n}} u(t) \nabla\left[F^{\prime} \circ u(t)-F^{\prime} \circ u_{D^{*}}\right] \cdot\left\{\nabla c^{*}\left[\nabla\left(F^{\prime} \circ u(t)\right)\right]+y\right\} \mathrm{d} y .
\end{aligned}
$$

The above energy dissipation follows using that $u_{D_{*}}$ satisfies $(1.12)$ and $\nabla c^{*} \circ \nabla c=$ id. This integration by parts can be justified using Lemma 2.3 by a standard argument introducing a cut-off function like in [6, Proposition 2.6]. Since the arguments are exactly equal, we do not perform any further details. By the convexity of $c^{*}$,

$$
\left[\nabla c^{*}(a)-\nabla c^{*}(b)\right] \cdot(a-b) \geq 0
$$

with equality if and only if $a=b$. So the Fisher information is non-negative and zero if and only if $u$ and $u_{D_{*}}$ have the same mass and such that $\nabla\left(F^{\prime} \circ u(\tau)-F^{\prime} \circ u_{D_{*}}\right)=0$, i.e. $u=u_{D_{*}}$.

With these ingredients, we can obtain a first result of convergence toward stationary states. 
Lemma 2.5 (Uniform convergence) Let $u \in C\left([0, \infty) ; L^{1}\left(\mathbb{R}^{n}\right)\right)$ be a solution of (1.9) for an initial data satisfying $(\mathrm{H} 1)$, then $\lim _{t \rightarrow \infty} w(t, x)=1$ uniformly in compact sets of $\mathbb{R}^{n}$.

Proof. Define $u^{h}(t, x):=u(h+t, x)$, for any given $h>0$ and $t \in[0,1]$. It is also well-known [13] that equi-bounded set of solutions of (1.1) are equi-continuous in time. This property carries over to $u(t)$ by the change of variables in (1.7). This fact together with the uniform bounds in $C^{1, \alpha}$ obtained in Lemma 2.2 and the Ascoli-Arzelá theorem implies that for any sequence $\left(h_{n}\right)_{n \in \mathbb{N}}$ there exists a sub-sequence $\left(h_{n}\right)_{n \in \mathbb{N}}$, denoted with the same index, such that $\left\{u^{h_{n}}\right\}_{n \in \mathbb{N}}$ converges to a function $u_{\infty}$ uniformly in compact sets of $[0,1] \times \mathbb{R}^{n}$, and moreover, $u_{\infty}(t) \in C^{1, \alpha}\left(\mathbb{R}^{n}\right)$ for all $t \in[0,1]$. Since $\mathcal{E}\left[u(t) \mid u_{D_{*}}\right]$ is non-increasing and positive it has a limit as $t \rightarrow \infty$ and

$$
\left.\int_{0}^{1} \mathcal{I}\left[u\left(s+h_{n}\right) \mid u_{D_{*}}\right] \mathrm{d} s=\int_{h_{n}}^{h_{n}+1} \mathcal{I}\left[u(s) \mid u_{D_{*}}\right] \mathrm{d} s=\mathcal{E}\left[u\left(h_{n}\right) \mid u_{D_{*}}\right]-\mathcal{E}\left[u\left(h_{n}+1\right)\right) \mid u_{D_{*}}\right] \rightarrow_{t \rightarrow \infty} 0,
$$

the function $t \mapsto \mathcal{I}\left[u^{h_{n}}(t) \mid u_{D_{*}}\right]$ is integrable on $[0,1]$ and converges to zero as $n \rightarrow \infty$. By (2.6), $\mathcal{I}$ is non-negative. By Fatou's lemma we have

$$
\int_{\mathbb{R}^{n}} \lim _{n \rightarrow \infty} u^{h_{n}}(t, x) \nabla\left(F^{\prime} \circ u^{h_{n}}(t, x)+c(x)\right) \cdot\left(\nabla c^{*}\left[\nabla F^{\prime} \circ u^{h_{n}}(t, x)+\nabla c^{*} \circ \nabla c(x)\right]\right) \mathrm{d} x=0 .
$$

As a consequence of $(2.6), u_{\infty}$ satisfies $\nabla\left[F^{\prime} \circ u_{\infty}(x)+c(x)\right]=0$, from which $u_{\infty}=u_{D}$ for some $D>0$. By conservation of mass $D=D_{*}$. Since the limit of all the convergent sub-sequences is uniquely determined by $u_{D_{*}}$, the result is proved.

Proposition 2.6 (Convergence in $L^{p}$-spaces) Let $u \in C\left([0, \infty) ; L^{1}\left(\mathbb{R}^{n}\right)\right)$ be a solution of the scaled doubly-nonlinear equation (1.9) for an initial data satisfying (H1), then

$$
\lim _{t \rightarrow \infty}\left\|u(t)-u_{D_{*}}\right\|_{p}=0 \quad \text { for any } p \in[1, \infty] \text { and } \lim _{t \rightarrow \infty} \mathcal{E}\left[u(t) \mid u_{D_{*}}\right]=0 .
$$

Proof. By Lemma 2.5, $\lim _{t \rightarrow \infty}\left|u(t, x)-u_{D_{*}}(x)\right|=0$ for any $x \in \mathbb{R}^{n}$. Moreover, by assumptions (H1), for $|x|$ large enough

$$
\left|u(t)-u_{D_{*}}\right| \leq \max \left\{\left|u_{D_{0}}-u_{D_{*}}\right|,\left|u_{D_{1}}-u_{D_{*}}\right|\right\}=O\left(|x|^{-q(2-\gamma) /(1-\gamma)}\right) .
$$

So the difference between $\left|u(t)-u_{D_{*}}\right|^{\theta}$ is in $L^{1}\left(\mathbb{R}^{n}\right)$ if $\theta>\Theta(p, m)$ with

$$
\Theta(p, m):=\frac{n(1-\gamma)}{q(2-\gamma)} .
$$

It is easy to check that $\Theta(p, m)$ is a decreasing function of $\gamma$ and so of $m$. Since $q>1$, in the range of exponents (1.5), we have

$$
\Theta(p, m) \leq \Theta\left(p, m_{c}(p)\right)=\frac{n}{n+q}<1 .
$$

By Lebesgue's dominated convergence theorem, it implies that $u(t)$ converges to $u_{D_{*}}$ in $L^{\theta}\left(\mathbb{R}^{n}\right)$, for any $\theta \in[1, \infty)$. Finally, we use the following interpolation lemma, due to Nirenberg, [30, p. 126]:

$$
\|f\|_{\infty} \leq C\|f\|_{C^{1}\left(\mathbb{R}^{n}\right)}^{\frac{n}{n+2}}\|f\|_{2}^{\frac{2}{n+2}} \quad \forall f \in C\left(\mathbb{R}^{n}\right),
$$


for $f=u(t)-u_{D_{*}}$ together with (2.4) to obtain the result in the uniform norm.

By Taylor's formula on the integrand of the relative entropy we have

$$
F \circ u(x)-F \circ u_{D_{*}}(x)-F^{\prime} \circ u_{D_{*}}(x)\left(u(x)-u_{D_{*}}(x)\right)=\frac{1}{2} F^{\prime \prime} \circ \xi(x)\left(u(x)-u_{D_{*}}(x)\right)^{2}
$$

with

$$
\left.u_{D_{*}}(x) W_{0} \leq \min \left(u(x), u_{D_{*}}(x)\right) \leq \xi(x) \leq \max \left(u(x), u_{D_{*}}(x)\right)\right) \leq u_{D_{*}}(x) W_{1},
$$

due to (H1), see also (H1'). For $x$ large enough

$$
C u_{D_{0}}^{\gamma-2}\left(U_{D_{1}}-U_{D_{0}}\right)^{2} \sim|x|^{-q(2-\gamma) /(1-\gamma)} .
$$

Since $\Theta(p, m)<1$, the integrand of the relative entropy is bounded by an integrable function. The convergence to 0 of the relative entropy thus holds by the Lebesgue dominated convergence theorem.

Remark 2.7 In contrast with [6], we do not generally have the convergence in $C^{1, \alpha}\left(\mathbb{R}^{n}\right)$.

\section{Linear stability}

To prove the decay (1.16) of $u(t)$ to $u_{D_{*}}$ in the energy form, it is sufficient to establish the following nonlinear Hardy-Poincaré type inequality:

$$
\mathcal{E}\left[u \mid u_{D^{*}}\right] \leq \frac{1}{\beta} \mathcal{I}\left[u \mid u_{D^{*}}\right],
$$

for some $\beta>0$ and $u \in C^{1, \alpha}\left(\mathbb{R}^{n}\right)$ verifying (H1). Indeed, (3.1) combined with Proposition 2.4 yield

$$
\frac{\mathrm{d}}{\mathrm{d} t} \mathcal{E}\left[u(t) \mid u_{D^{*}}\right] \leq-\beta \mathcal{E}\left[u(t) \mid u_{D^{*}}\right]
$$

and this leads to the energy decay (1.16) by a simple Gronwall argument. To prove (3.1), we will first show a linearised version of this inequality - i.e., a weighted Poincaré inequality -, by considering the perturbation $u(t)=u_{D_{*}}+\epsilon v(t)$ of the solution $u(t)$ to (1.9). This will lead to the convergence of $v(t)$ to 0 in relative entropy for the linearised equation of (1.9), as we will show below. Next section will be devoted to compare the relative entropy and Fisher information in (3.1) with their linearised analogues.

For clarity in our exposition, let us start by formally deriving the form of the weighted Poincaré inequality that we will be dealing with below. Using the perturbation $u=u_{D^{*}}+\epsilon v$ and the second order Taylor expansion of $F\left(u_{D^{*}}+\epsilon v\right)$ at $\epsilon=0$ on the expression of the relative entropy (1.15), we have that

$$
F \circ u-F \circ u_{D^{*}}=\epsilon v F^{\prime}\left(u_{D^{*}}\right)+\frac{\epsilon^{2}}{2} v^{2} F^{\prime \prime} \circ u_{D^{*}}+O\left(\epsilon^{3}\right),
$$

and then $\mathcal{E}\left[u \mid u_{D^{*}}\right]$ linearises as:

$$
\mathcal{E}\left[u \mid u_{D^{*}}\right]=\frac{\epsilon^{2}}{2} \int_{\mathbb{R}^{n}} v^{2} F^{\prime \prime} \circ u_{D^{*}}+O\left(\epsilon^{3}\right) .
$$


Let us hence introduce the weighted $L^{2}$-norm:

$$
\mathrm{E}[v]=\frac{1}{2} \int_{\mathbb{R}^{n}} v^{2}(x) F^{\prime \prime} \circ u_{D^{*}}(x) \mathrm{d} x,
$$

which will play the role of the linearised relative entropy.

Concerning the linearisation of the Fisher information, from the first order Taylor expansion of $F^{\prime} \circ u=F^{\prime}\left(u_{D_{*}}+\epsilon v\right)$ at $\epsilon=0$, we have

$$
B:=\nabla\left[F^{\prime} \circ u\right]=A+\epsilon W+O\left(\epsilon^{2}\right) .
$$

with

$$
A:=\nabla\left[F^{\prime} \circ u_{D^{*}}\right]=\nabla c \text { and } W:=\nabla\left[v F^{\prime \prime} \circ u_{D^{*}}\right] .
$$

Then using that $\nabla c^{*}(z)=z|z|^{p-2}$, we obtain

$$
\nabla c^{*}(B)=\nabla c^{*}(A)+\epsilon|A|^{p-2} W+\epsilon(p-2)|A|^{p-4}(A \cdot W) A+O\left(\epsilon^{2}\right) .
$$

Combining (3.3) and (3.5), we see that $\mathcal{I}\left[u \mid u_{D^{*}}\right]$ formally linearises as:

$$
\mathcal{I}\left[u \mid u_{D^{*}}\right]=\epsilon^{2} \int_{\mathbb{R}^{n}} u_{D^{*}}|A|^{p-2}|W|^{2} \mathrm{~d} x+\epsilon^{2}(p-2) \int_{\mathbb{R}^{n}} u_{D^{*}}|A|^{p-4}(A \cdot W)^{2} \mathrm{~d} x+O\left(\epsilon^{3}\right) .
$$

Hence, for $\epsilon$ small enough, the nonlinear Hardy-Poincaré inequality (3.1) linearises as

$$
\begin{aligned}
\frac{\beta}{2} \int_{\mathbb{R}^{n}} v^{2} F^{\prime \prime} \circ u_{D^{*}} \leq & \int_{\mathbb{R}^{n}} u_{D^{*}}|\nabla c|^{p-2}\left|\nabla\left[v F^{\prime \prime} \circ u_{D^{*}}\right]\right|^{2} \mathrm{~d} x \\
& +(p-2) \int_{\mathbb{R}^{n}} u_{D^{*}}|\nabla c|^{p-4}\left[\nabla c \cdot \nabla\left(v F^{\prime \prime} \circ u_{D^{*}}\right)\right]^{2} \mathrm{~d} x .
\end{aligned}
$$

It will be shown below that the l.h.s of (3.6) is a Lyapunov function - and the relative entropy - for the linearised equation of (1.9), and the r.h.s of (3.6) corresponds to the dissipation of this relative entropy, up to a constant.

Let $u$ be the solution of (1.9), and consider the small perturbation

$$
u(t)=u_{D^{*}}+\epsilon v(t)
$$

of $u_{D^{*}}$, where $\epsilon>0$ is small, and $v(t) \in C^{1, \alpha}\left(\mathbb{R}^{n}\right)$ for some $\alpha \in(0,1)$. Because of the massconservation (2.1)-(2.2), we have using the rescaling (1.7)-(1.8), that

$$
\int_{\mathbb{R}^{n}} v(t, x) \mathrm{d} x=0, \quad \forall t \geq 0
$$

Moreover (3.7) implies that

$$
\frac{\partial u}{\partial t}=\epsilon \frac{\partial v}{\partial t}
$$

On the other hand, using (1.12), we have that $\nabla c^{*}(A)=\nabla c^{*}[-\nabla c(x)]=-x$, and then (3.5) gives that

$$
u\left[\nabla c^{*}(B)+x\right]=\epsilon u_{D^{*}}\left[|A|^{p-2} W+(p-2)|A|^{p-4}(A \cdot W) W\right]+O\left(\epsilon^{2}\right) .
$$


Inserting (3.8)-(3.9) into (1.9), we formally obtain after simplifying by $\epsilon$ and then setting $\epsilon=0$, that the linearised problem to $(1.9)$ is:

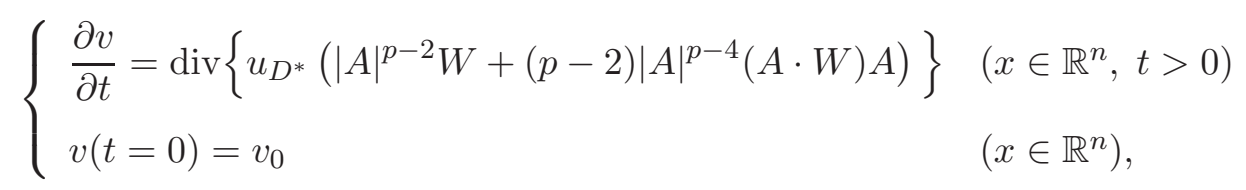

with $v_{0} \in L^{1}\left(\mathbb{R}^{n}\right)$ of zero average. We can easily check that Eq. (3.10) has the linearised relative entropy (3.2) as Lyapunov functional. Actually, differentiating $\mathrm{E}[v(t)]$ along a solution $v$ of (3.10), we formally have by a straightforward computation, that

$$
\frac{\mathrm{d}}{\mathrm{d} t} \mathrm{E}[v(t)]=-\left(\mathbf{I}[v(t)]+(p-2) \mathbf{I}_{0}[v(t)]\right)
$$

where

$$
\mathrm{I}[v(t)]=\int_{\mathbb{R}^{n}}|W(t)|^{2} u_{D^{*}}|A|^{p-2} \mathrm{~d} x \quad \text { and } \quad \mathrm{I}_{0}[v(t)]=\int_{\mathbb{R}^{n}}(A \cdot W(t))^{2} u_{D^{*}}|A|^{p-4} \mathrm{~d} x .
$$

The Cauchy-Schwarz inequality implies that $|A|^{p-4}(A \cdot W(t)) \leq|A|^{p-2}|W(t)|^{2}$, and as a consequence, $\mathrm{I}_{0}[v(t)] \leq \mathrm{I}[v(t)]$. Using $1<p<2$, we have

$$
\mathbf{I}[v(t)]+(p-2) \mathbf{I}_{0}[v(t)] \geq(p-1) \mathbf{I}[v(t)] \geq 0 .
$$

In case $p>2$, it is direct that $\mathbf{I}[v(t)]+(p-2) \mathbf{I}_{0}[v(t)] \geq \mathbf{I}[v(t)] \geq 0$. From these estimates, the dissipation (3.11) and $|A(x)|=\left|\nabla\left[F^{\prime} \circ u_{D^{*}}\right](x)\right|>0$ for all $x \in \mathbb{R}^{n}$, we readily formally conclude that the unique steady state is the zero solution.

The objective of the rest of this section is to show the following asymptotic exponential relaxation of the linearised equation (3.10):

Theorem 3.1 Let $m$ satisfying (1.5) and $v_{0} \in L^{1}\left(\mathbb{R}^{n}\right)$ with zero average. Consider $v(t)$ the solution to (3.10) with initial data $v_{0}$. There exists a constant $\beta>0$ such that

$$
E[v(t)] \leq e^{-\beta t} E\left[v_{0}\right]
$$

Let us concentrate first in the case $1<p<2$. To derive this exponential rate of convergence, we establish the following functional inequality

$$
\mathrm{E}[v] \leq \frac{1}{\beta}\left(\mathrm{I}[v]+(p-2) \mathrm{I}_{0}[v]\right), \quad \beta>0,
$$

for all $v \in C^{1, \alpha}\left(\mathbb{R}^{n}\right)$ with zero average. This inequality corresponds to the formal linearisation (3.6) of the nonlinear Hardy-Poincaré inequality (3.1). In fact, because of (3.12), it is sufficient to prove the following weighted Poincaré type inequality:

$$
\mathrm{E}[v] \leq \frac{p-1}{\beta} \mathrm{I}[v], \quad \beta>0
$$

for all $v \in C^{1, \alpha}\left(\mathbb{R}^{n}\right)$ with zero average. This is equivalent to show the Hardy-Poincaré type inequality:

$$
\int_{\mathbb{R}^{n}} g^{2} \mathrm{~d} \mu(x) \leq \tilde{\beta} \int_{\mathbb{R}^{n}}|\nabla g|^{2} \mathrm{~d} \nu(x)
$$


for some $\tilde{\beta}>0$, where $g$ is any function satisfying $\int_{\mathbb{R}^{n}} g \mathrm{~d} \mu(x)=0$, and

$$
\mathrm{d} \mu(x)=\frac{\mathrm{d} x}{F^{\prime \prime} \circ u_{D^{*}}(x)}, \quad \mathrm{d} \nu(x)=u_{D^{*}}(x)\left|\nabla\left(F^{\prime} \circ u_{D^{*}}(x)\right)\right|^{p-2} \mathrm{~d} x .
$$

Note that the functions $v$ and $g$ in (3.14)-(3.15) are related by $g=v F^{\prime \prime} \circ u_{D^{*}}$, and $\tilde{\beta}=2(p-1) / \beta$. The inequality (3.15) is also enough to prove the needed inequality in the case $p>2$ since (3.11) implies that

$$
\frac{\mathrm{d}}{\mathrm{d} t} \mathrm{E}[v(t)] \leq-\mathrm{I}[v(t)]
$$

Lemma 3.2 (Hardy-Poincaré type inequality) Let $m, n, p$ be such that $1<p<\infty$ and $m_{c}(p)<m<\frac{n-p+1}{n(p-1)}$. Then, there exits a constant $\tilde{\beta}>0$ such that

$$
\int_{\mathbb{R}^{n}} g^{2} \mathrm{~d} \mu(x) \leq \tilde{\beta} \int_{\mathbb{R}^{n}}|\nabla g|^{2} \mathrm{~d} \nu(x),
$$

for any function $g \in C^{1, \tilde{\alpha}}\left(\mathbb{R}^{n}\right)$ satisfying $\int_{\mathbb{R}^{n}} g \mathrm{~d} \mu(x)=0$ with $0<\tilde{\alpha}<1$, where $\mu$ and $\nu$ are defined by (3.16).

We keep calling this inequality, "Hardy-Poincaré inequality" to remind the link with the inequality proved in $[5,6]$, but here we only use the Poincaré type part of the inequality. The proof of the Hardy-Poincaré inequality was performed in $[5,6]$ and an estimate of the constant $\tilde{\beta}$ is also established. This proof can be adapted to Lemma 3.2. For completeness, we give here the proof of this variant of the Hardy-Poincaré inequality.

Proof. We first observe that we can reduce to show the inequality for the Schwartz class $g \in \mathcal{D}\left(\mathbb{R}^{n}\right)$ by simple approximation arguments. In $L^{2}\left(\mathbb{R}^{n}, \mathrm{~d} \mu\right)$ we consider the closable quadratic form $v \mapsto \mathcal{Q}(v):=\int_{\mathbb{R}^{n}}|\nabla g|^{2} \mathrm{~d} \nu$ and $-\mathcal{L}$ the unique non-negative, self-adjoint operator in $L^{2}\left(\mathbb{R}^{n}, \mathrm{~d} \mu\right)$ associated with the closure of $\mathcal{Q}$. By Persson's theorem [32]

$$
\inf \sigma_{\text {ess }}(-\mathcal{L})=\lim _{R \rightarrow \infty} \inf _{v \in \mathcal{H}_{R}} \frac{\mathcal{Q}(v)}{\int_{\mathbb{R}^{n}}|g|^{2} \mathrm{~d} \mu}
$$

where $\mathcal{H}_{R}:=\left\{v \in H^{1}\left(\mathbb{R}^{d}, \mathrm{~d} \nu\right): \operatorname{supp}(v) \subset \mathbb{R}^{n} \backslash B(0, R)\right\}$. Roughly speaking it means that the inequality is true for any weights with the same behaviour in a neighbourhood of $+\infty$. By a straightforward computation using (1.12) and (1.11), we have that

$$
\mathrm{d} \mu(x)=\frac{1}{m}\left(D_{*}+\frac{1-\gamma}{m q}|x|^{q}\right)^{\frac{2-\gamma}{\gamma-1}} \mathrm{~d} x \sim|x| \rightarrow \infty \frac{1}{m}\left(\frac{1-\gamma}{m q}\right)^{\frac{2-\gamma}{\gamma-1}}|x|^{2 \alpha-2} \mathrm{~d} x
$$

and

$$
\mathrm{d} \nu(x)=|x|^{2-q}\left(D_{*}+\frac{1-\gamma}{m q}|x|^{q}\right)^{\frac{1}{\gamma-1}} \mathrm{~d} x \sim_{|x| \rightarrow \infty}\left(\frac{1-\gamma}{m q}\right)^{\frac{1}{\gamma-1}}|x|^{2 \alpha} \mathrm{d} x
$$

with $\alpha$ chosen in such a way that $q(2-\gamma) /(\gamma-1)=2(\alpha-1)$ and $2-q+q /(\gamma-1)=2 \alpha$, that is,

$$
\alpha=1+\frac{q(2-\gamma)}{2(\gamma-1)} \quad \text { or equivalently } \quad \alpha=\frac{2-q}{2}+\frac{q}{2(\gamma-1)} .
$$


It is left to the reader to check that $\alpha<-(d-2) / 2$ in the range of $1<p<\infty$ and $m_{c}(p)<m$, and thus, we can apply [5, Theorem 1] to obtain

$$
\inf \sigma_{\mathrm{ess}}(-\mathcal{L}) \geq \frac{1-\gamma}{q} \kappa_{\alpha}
$$

where $\kappa_{\alpha}$ is the constant of the following Hardy inequality with weight, see also [6]. We refer to [5, Theorem 1] for estimates on the constant $\kappa_{\alpha}$ depending of $-n<\alpha$ or $\alpha \leq-n$, see also [6]. We remark that both cases happen depending on the precise values of $m_{c}(p)<m<\frac{n-p+1}{n(p-1)}$ and $1<p<\infty$.

The lowest eigenvalue of $-\mathcal{L}$ is $\lambda_{1}=0$ with eigenfunctions given by the constants functions. Zero mean-value solutions belong to the orthogonal set to the eigenspace associated to $\lambda_{1}$. Since $\lambda_{1}$ is non-degenerate we obtain the desired result for some $\lambda_{2} \in\left(0, \kappa_{\alpha}\right]$.

Since the only behaviour of the weights that counts is their growth at infinity, we can avoid the singularity of the weight at the origin for the singular case $1<p<2$ directly to obtain the following stronger inequality.

Corollary 3.3 For any $\epsilon>0$, there exists a constant $\tilde{\beta}_{\epsilon}>0$ such that

$$
\int_{\mathbb{R}^{n}} g^{2} \mathrm{~d} \mu(x) \leq \tilde{\beta}_{\epsilon} \int_{\mathbb{R}^{n}}|\nabla g|^{2} \mathrm{~d} \nu_{\epsilon}(x),
$$

for any function $g \in C^{1, \alpha}\left(\mathbb{R}^{n}\right)$ satisfying $\int_{\mathbb{R}^{n}} g \mathrm{~d} \mu(x)=0$, where $\mu$ is defined by (3.16), and

$$
\mathrm{d} \nu_{\epsilon}(x)=u_{D^{*}}(x)\left(\epsilon+\left|\nabla\left(F^{\prime} \circ u_{D^{*}}(x)\right)\right|\right)^{p-2} \mathrm{~d} x .
$$

Therefore, setting $v=g /\left(F^{\prime \prime} \circ u_{D^{*}}\right)$, we have the stronger weighted Poincaré inequality

$$
E[v] \leq \frac{\tilde{\beta}_{\epsilon}}{2} I^{(\epsilon)}[v]
$$

for all $v \in C^{1, \tilde{\alpha}}\left(\mathbb{R}^{n}\right)$ with zero average and $0<\tilde{\alpha}<1$, where

$$
I^{(\epsilon)}[v]=\int_{\mathbb{R}^{n}}\left|\nabla\left(v F^{\prime \prime} \circ u_{D^{*}}\right)\right|^{2}\left(\epsilon+\left|\nabla\left(F^{\prime} \circ u_{D^{*}}\right)\right|\right)^{p-2} u_{D^{*}} \mathrm{~d} x .
$$

Remark 3.4 The Bakry-Emery approach used in [10] to establish the weighted Poincaré inequality inequality (3.14) when $p=2$, does not seem to apply here when $1<p<2$. For illustration, let us consider the particular case $m=n=1$, that is the linearisation of the 1-dimensional rescaled $p$-Laplacian equation, $\partial_{t} v=\operatorname{div}\left\{(p-1) u_{D^{*}}|A|^{p-2} W\right\}$, where $A$ and $W$ are defined by (3.4). In this case, the relative entropy dissipation equation (3.11) simplifies as

$$
\frac{\mathrm{d} E[v(t)]}{\mathrm{d} t}=-(p-1) I[v(t)]
$$

and it is easy to show that its dissipation is

$$
-\frac{\mathrm{d} \boldsymbol{l}[v(t)]}{\mathrm{d} t}=2(p-1)^{2} \mathcal{D}\left[v(t) \mid v_{D^{*}}\right],
$$


where

$$
\mathcal{D}\left[v(t) \mid v_{D^{*}}\right]=\int_{\mathbb{R}^{n}} F^{\prime \prime} \circ u_{D^{*}}\left[\operatorname{div}\left(u_{D^{*}}|A|^{p-2} W\right)\right]^{2} \mathrm{~d} x .
$$

Following [10], if one can establish the estimate $\mathcal{D}\left[v(t) \mid v_{D^{*}}\right] \geq \lambda /\left[v(t) \mid v_{D^{*}}\right]$ for some $\lambda>0$, then it will imply the weighted Poincaré inequality (3.14). But by a direct computation, we can show that

$$
\begin{aligned}
\mathcal{D}\left[v(t) \mid v_{D^{*}}\right]= & \left(1+\frac{(p-2)^{2}}{p(p-1)}\right) I\left(v(t) \mid v_{D^{*}}\right)+\int_{\mathbb{R}^{n}} F^{\prime \prime} \circ u_{D^{*}}\left(u_{D^{*}}|A|^{p-2} \operatorname{div} W\right)^{2} \mathrm{~d} x \\
& +K \frac{p-2}{(p-1)^{2}} \int_{\mathbb{R}^{n}} u_{D^{*}}|A|^{p-2} W^{2}|y|^{-q} \mathrm{~d} x .
\end{aligned}
$$

Note that the second term in the above expression is non-negative (because $F$ is convex), while the last term is non-positive in the range $1<p<2$. If $p \geq 2$, the last term is also non-negative and we obtain $\mathcal{D}\left(v(t) \mid v_{D^{*}}\right) \geq I\left(v(t) \mid v_{D^{*}}\right)$, that is $\lambda=1$. This then yields the desired inequality (3.14) when $p \geq 2$. But if $1<p<2$, one cannot derive this estimate from (3.19), at least at cursory glance.

Proof of Theorem 3.1 We apply Lemma 3.2 to $g(t)=v(t) F^{\prime \prime}$ ou $_{D^{*}}$ and $\beta=2(p-1) / \tilde{\beta}$. By $(3.11)$ and (3.12) we have

$$
\frac{\mathrm{dE}[u(t)]}{\mathrm{d} t} \leq-\beta \mathrm{E}[v(t)]
$$

This leads to (3.13) by a Gronwall estimate.

\section{Nonlinear stability}

The first step to go from linear to nonlinear stability is to use that our solution is sandwiched between two Barenblatt profiles to compare the nonlinear relative entropy and its dissipation with their linearised counterparts.

Proposition 4.1 (Comparison linear/nonlinear relative entropy) Consider a function $u$ satisfying (H1). Then there exist positive constants $C_{1}$ and $C_{2}$ such that

$$
C_{1} E\left[u-u_{D_{*}}\right] \leq \mathcal{E}\left[u \mid u_{D_{*}}\right] \leq C_{2} E\left[u-u_{D_{*}}\right] .
$$

Proof. The asserted result follows from the end of the proof of Proposition 2.6 with $C_{1}:=m W_{0}^{\gamma-2}$ and $C_{2}:=m W_{1}^{\gamma-2}$.

The next objective is to compare the nonlinear Fisher information, $\mathcal{I}\left[u(t) \mid u_{D^{*}}\right]$, with its linear analogue, I $\left[u(t)-u_{D^{*}}\right]$ along solutions of (1.9). Let us point out that the weight

$$
\left|\nabla\left(F^{\prime} \circ u_{D_{*}}\right)\right|^{p-2}=|x|^{2-q}
$$

in the linearised entropy dissipation diverges at the origin for $1<p<2$. This singular behaviour makes complicated any attempt to compare it with nonlinear Fisher analogues. Due to the singularity of the weight $\left|\nabla\left(F^{\prime} \circ u_{D^{*}}\right)\right|^{p-2}$ at $x=0$, we will replace I $\left[u(t)-u_{D^{*}}\right]$ by its regularised analogue $\mathbf{I}^{(\epsilon)}\left[u(t)-u_{D^{*}}\right]$ defined by (3.18), where $\epsilon>0$ is a fixed constant. 
Proposition 4.2 (Comparison linear/non-linear Fisher information) Assume that $u$ is the solution of (1.9), and set $v=u-u_{D^{*}}$. Then:

1. Case $1<p<2$ : Given $\epsilon>0$, there exist $t_{0}>0$ and positive constants $\kappa_{1}$ and $\kappa_{2}$ such that for all $t>t_{0}$,

$$
I^{(\epsilon)}[v(t)] \leq \kappa_{1} \mathcal{I}\left[u(t) \mid u_{D^{*}}\right]+\kappa_{2} E[v(t)] .
$$

2. Case $p>2$ : There exist $t_{0}>0$ and positive constants $\kappa_{1}$ and $\kappa_{2}$ such that for all $t>t_{0}$,

$$
I[v(t)] \leq \kappa_{1} \mathcal{I}\left[u(t) \mid u_{D^{*}}\right]+\kappa_{2} E[v(t)] .
$$

Moreover $\kappa_{2}$ can be chosen arbitrary small provided that $t_{0}$ is large enough.

The proof of this proposition is organised as follows:

Claim 1: We first show that, for all $\epsilon \geq 0$ and $1<p<2$ and for $\epsilon=0$ if $p \geq 2$, there exists $\kappa_{0}>0$ such that

$$
\mathbf{I}^{(\epsilon)}[v(t)] \leq \kappa_{0} \mathbf{I}_{\gamma}^{(\epsilon)}[v(t)]+\kappa_{2} \mathrm{E}[v(t)]
$$

where

$$
\mathrm{I}_{\gamma}^{(\epsilon)}[v]=\int_{\mathbb{R}^{n}}\left|\nabla\left[F^{\prime} \circ u-F^{\prime} \circ u_{D^{*}}\right]\right|^{2}\left(\epsilon+\left|\nabla\left(F^{\prime} \circ u_{D^{*}}(x)\right)\right|\right)^{p-2} u_{D^{*}} \mathrm{~d} x .
$$

Claim 2: Next we show that if $1<p<2$, then for all $\epsilon>0$ there exists $\delta>0$ such that

$$
\mathrm{I}_{\gamma}^{(\epsilon)}[v(t)] \leq \delta \mathcal{I}\left[u(t) \mid u_{D^{*}}\right]
$$

whereas if $2<p<\infty$, then there exists $\delta>0$ such that

$$
\mathrm{I}_{\gamma}[v(t)] \leq \delta \mathcal{I}\left[u(t) \mid u_{D^{*}}\right]
$$

Combining (4.3) and (4.5)-(4.6), we obtain the desired inequalities (4.1)-(4.2) with $\kappa_{1}=\delta \kappa_{0}$; here $\mathrm{I}_{\gamma}=\mathrm{I}_{\gamma}^{(0)}$ and $\mathbf{I}=\mathrm{I}^{(0)}$.

Proof of Claim 1: Here we follow the arguments of the proof of Lemma 5.1 in [6]. Indeed, let $h_{k}(w)=\left(w^{k-1}-1\right) /(k-1)$, where

$$
w(t, x)=\frac{u(t, x)}{u_{D^{*}}(x)} .
$$

Because of assumption (H1), we have that $W_{0} \leq w(t, x) \leq W_{1}$, where the constant $W_{0}$ and $W_{1}$ are such that $0<W_{0}<1<W_{1}$. By studying the function $h_{2} / h_{\gamma}$ on $\left[W_{0}, W_{1}\right]$, we have

$$
\alpha_{0} h_{\gamma}(w)^{2} \leq h_{2}(w)^{2} \leq \alpha_{1} h_{\gamma}(w)^{2},
$$

and

$$
h_{2}^{\prime}(w)^{2} \leq \alpha_{2} h_{\gamma}^{\prime}(w)^{2},
$$

where

$$
\alpha_{0}:=|\gamma-1|^{2}\left|\frac{W_{0}-1}{W_{0}^{\gamma-1}-1}\right|^{2}<1, \quad \alpha_{1}:=|\gamma-1|^{2}\left|\frac{W_{1}-1}{W_{1}^{\gamma-1}-1}\right|^{2}>1
$$


and

$$
\alpha_{2}:=W_{1}^{2(2-\gamma)}>1
$$

Now, define

$$
\mathbf{I}_{k}^{(\epsilon)}[v]:=m^{2} \int_{\mathbb{R}^{n}}\left|\nabla\left(u_{D^{*}}^{\gamma-1} h_{k}(w)\right)\right|^{2}\left(\epsilon+|x|^{q-1}\right)^{p-2} u_{D^{*}} \mathrm{~d} x .
$$

We have that $\mathbf{I}^{(\epsilon)}=\mathbf{I}_{2}^{(\epsilon)}$ and for $k=\gamma, \mathbf{I}_{\gamma}^{(\epsilon)}$ is defined in (4.4). Next we compute $\mathbf{I}_{k}^{(\epsilon)}[v]$. By expanding $\left|\nabla\left(u_{D^{*}}^{\gamma-1} h_{k}(w)\right)\right|^{2}$, we have

$$
\begin{aligned}
\mathrm{I}_{k}^{(\epsilon)}[v]= & m^{2} \int_{\mathbb{R}^{n}} u_{D^{*}}^{2 \gamma-1} h_{k}^{\prime}(w)^{2}|\nabla w|^{2}\left(\epsilon+|x|^{q-1}\right)^{p-2} \mathrm{~d} x \\
& +(1-\gamma)^{2} \int_{\mathbb{R}^{n}} h_{k}(w)^{2}|x|^{2(q-1)}\left(\epsilon+|x|^{q-1}\right)^{p-2} u_{D^{*}} \mathrm{~d} x \\
& +2 m(1-\gamma) \int_{\mathbb{R}^{n}} u_{D^{*}}^{\gamma} h_{k}^{\prime}(w) h_{k}(w)|x|^{q-2}\left(\epsilon+|x|^{q-1}\right)^{p-2} \nabla w \cdot x \mathrm{~d} x .
\end{aligned}
$$

Integrating by parts, the last integral can be rewritten as

$$
\begin{aligned}
& \int_{\mathbb{R}^{n}} u_{D^{*}}^{\gamma} h_{k}^{\prime}(w) h_{k}(w)|x|^{q-2}\left(\epsilon+|x|^{q-1}\right)^{p-2} \nabla w \cdot x \mathrm{~d} x \\
&=\frac{1}{2} \int_{\mathbb{R}^{n}} \nabla\left(h_{k}(w)^{2}\right) \cdot|x|^{q-2} x\left(\epsilon+|x|^{q-1}\right)^{p-2} u_{D^{*}}^{\gamma} \mathrm{d} x \\
&=-\frac{1}{2} \int_{\mathbb{R}^{n}} h_{k}(w)^{2} \operatorname{div}\left(|x|^{q-2} x\left(\epsilon+|x|^{q-1}\right)^{p-2} u_{D^{*}}^{\gamma}\right) \mathrm{d} x \\
&=-\frac{1}{2} \int_{\mathbb{R}^{n}} h_{k}(w)^{2} \operatorname{div}\left(|x|^{q-2} x\left(\epsilon+|x|^{q-1}\right)^{p-2}\right) u_{D^{*}}^{\gamma} \mathrm{d} x \\
&+\frac{\gamma}{2 m} \int_{\mathbb{R}^{n}} h_{k}(w)^{2}|x|^{2(q-1)}\left(\epsilon+|x|^{q-1}\right)^{p-2} u_{D^{*}} \mathrm{~d} x .
\end{aligned}
$$

Then, for $\epsilon \geq 0$ if $1<p<2$ and $\epsilon=0$ if $p>2$,

$$
\begin{aligned}
\mathrm{I}_{k}^{(\epsilon)}[v]= & m^{2} \int_{\mathbb{R}^{n}} u_{D^{*}}^{2 \gamma-1} h_{k}^{\prime}(w)^{2}|\nabla w|^{2}\left(\epsilon+|x|^{q-1}\right)^{p-2} \mathrm{~d} x \\
& +(1-\gamma) \int_{\mathbb{R}^{n}} h_{k}(w)^{2}|x|^{2(q-1)}\left(\epsilon+|x|^{q-1}\right)^{p-2} u_{D^{*}} \mathrm{~d} x \\
& -m(1-\gamma) \int_{\mathbb{R}^{n}} h_{k}(w)^{2} \operatorname{div}\left(|x|^{q-2} x\left(\epsilon+|x|^{q-1}\right)^{p-2}\right) u_{D^{*}}^{\gamma} \mathrm{d} x
\end{aligned}
$$

Next we set $\kappa_{0}:=\max \left(\alpha_{1}, \alpha_{2}\right)$. Moreover, since $w$ uniformly converges to 1 as $t$ goes to $\infty$, then $\alpha_{0}, \alpha_{1}, \alpha_{2}$ and $\kappa_{0}>1$ can be chosen arbitrary close to 1 provided that $t>t_{0}$, for some $t_{0}$ large 
enough. Combining (4.7)-(4.8), we have

$$
\begin{aligned}
\mathrm{I}^{(\epsilon)}[v]=\mathrm{I}_{2}^{(\epsilon)}[v] \leq & m^{2} \alpha_{2} \int_{\mathbb{R}^{n}} u_{D^{*}}^{2 \gamma-1} h_{\gamma}^{\prime}(w)^{2}|\nabla w|^{2}\left(\epsilon+|x|^{q-1}\right)^{p-2} \mathrm{~d} x \\
& +\alpha_{1}(1-\gamma) \int_{\mathbb{R}^{n}} h_{\gamma}(w)^{2}|x|^{2(q-1)}\left(\epsilon+|x|^{q-1}\right)^{p-2} u_{D^{*}} \mathrm{~d} x \\
& -m(1-\gamma) \int_{\mathbb{R}^{n}} h_{2}(w)^{2} \operatorname{div}\left(|x|^{q-2} x\left(\epsilon+|x|^{q-1}\right)^{p-2}\right) u_{D^{*}}^{\gamma} \mathrm{d} x \\
\leq \kappa_{0} \mathbf{I}_{\gamma}^{(\epsilon)}[v] & +m(1-\gamma) \int_{\mathbb{R}^{n}} u_{D^{*}}^{\gamma} \operatorname{div}\left(|x|^{q-2} x\left(\epsilon+|x|^{q-1}\right)^{p-2}\right)\left(\kappa_{0} h_{\gamma}(w)^{2}-h_{2}(w)^{2}\right) \mathrm{d} x .
\end{aligned}
$$

The integration by parts is valid as

$$
h_{\gamma}(w)^{2}|x| u_{D^{*}}^{\gamma} \sim h_{2}(w)^{2}|x| u_{D^{*}}^{\gamma} \sim\left(U_{D_{0}}-U_{D_{1}}\right)^{2}|x| U_{D^{*}}^{\gamma-2} \sim|x|^{-q(2-\gamma) /(1-\gamma)+1-n}
$$

Finally, we observe that $0 \leq \kappa_{0} h_{\gamma}(w)^{2}-h_{2}(w)^{2} \leq\left(\kappa_{0} / \alpha_{0}-1\right) h_{2}(w)^{2}$ and by direct computation

$$
\left|\operatorname{div}\left(|x|^{q-2} x\left(\epsilon+|x|^{q-1}\right)^{p-2}\right)\right| \leq n+2(q-2),
$$

which is valid for $\epsilon \geq 0$ if $1<p<2$ and $\epsilon=0$ if $p>2$. We then deduce that

$$
\mathbf{I}^{(\epsilon)}[v(t)] \leq \kappa_{0} \mathbf{I}_{\gamma}^{(\epsilon)}[v(t)]+\kappa_{2} \mathrm{E}[v(t)]
$$

with $\kappa_{2}:=2\left(\kappa_{0} / \alpha_{0}-1\right)(1-\gamma)(n+2(q-2))>0$. Clearly $\kappa_{2}$ is arbitrary small provided that $t_{0}$ is large enough since $\kappa_{0} / \alpha_{0}=\max \left(\alpha_{1} / \alpha_{0}, \alpha_{2} / \alpha_{0}\right)>1$ gets arbitrarily close to 1 .

Proof of Claim $2-\left(\right.$ Case 1: $1<p<2$ ): First we expand $\mathbf{I}_{\gamma}^{(\epsilon)}[v(t)]$ and $\mathcal{I}\left[u(t) \mid u_{D^{*}}\right]$, and we have that

$$
\begin{aligned}
\mathbf{I}_{\gamma}^{(\epsilon)}[v(t)]= & \int_{\mathbb{R}^{n}}\left|\nabla\left[F^{\prime} \circ u\right]\right|^{2}\left(\epsilon+\left|\nabla\left[F^{\prime} \circ u_{D^{*}}\right]\right|\right)^{p-2} u_{D^{*}} \mathrm{~d} x \\
& +\int_{\mathbb{R}^{n}}\left|\nabla\left[F^{\prime} \circ u_{D^{*}}\right]\right|^{2}\left(\epsilon+\left|\nabla\left[F^{\prime} \circ u_{D^{*}}\right]\right|\right)^{p-2} u_{D^{*}} \mathrm{~d} x \\
& -2 \int_{\mathbb{R}^{n}} \nabla\left[F^{\prime} \circ u\right] \cdot \nabla\left[F^{\prime} \circ u_{D^{*}}\right]\left(\epsilon+\left|\nabla\left[F^{\prime} \circ u_{D^{*}}\right]\right|\right)^{p-2} u_{D^{*}} \mathrm{~d} x
\end{aligned}
$$

and

$$
\begin{aligned}
\mathcal{I}\left[u(t) \mid u_{D^{*}}\right]= & \int_{\mathbb{R}^{n}}\left|\nabla\left[F^{\prime} \circ u\right]\right|^{p} u \mathrm{~d} x+\int_{\mathbb{R}^{n}}\left|\nabla\left[F^{\prime} \circ u_{D^{*}}\right]\right|^{p} u \mathrm{~d} x \\
& -\int_{\mathbb{R}^{n}} \nabla\left[F^{\prime} \circ u\right] \cdot \nabla c^{*}\left(\nabla\left[F^{\prime} \circ u_{D^{*}}\right]\right) u \mathrm{~d} x \\
& -\int_{\mathbb{R}^{n}} \nabla\left[F^{\prime} \circ u_{D^{*}}\right] \cdot \nabla c^{*}\left(\nabla\left[F^{\prime} \circ u\right]\right) u \mathrm{~d} x .
\end{aligned}
$$

Next we use Young inequality $a \cdot b \leq c(a)+c^{*}(b)$ with $c(z)=|z|^{q} / q, a=\nabla c^{*}\left(\nabla\left[F^{\prime} \circ u\right]\right)$ and $b=\nabla\left[F^{\prime} \circ u_{D^{*}}\right]$, to have that

$$
\nabla\left[F^{\prime} \circ u_{D^{*}}\right] \cdot \nabla c^{*}\left(\nabla F^{\prime}(u)\right) \leq \frac{1}{q}\left|\nabla\left[F^{\prime} \circ u\right]\right|^{p}+\frac{1}{p}\left|\nabla\left[F^{\prime} \circ u_{D^{*}}\right]\right|^{p},
$$


Then $\mathcal{I}\left[u(t) \mid u_{D^{*}}\right]$ can be estimated as

$$
\begin{aligned}
\mathcal{I}\left[u(t) \mid u_{D^{*}}\right] \geq & \frac{1}{p} \int_{\mathbb{R}^{n}}\left|\nabla\left[F^{\prime} \circ u\right]\right|^{p} u \mathrm{~d} x+\frac{1}{q} \int_{\mathbb{R}^{n}}\left|\left(\nabla\left[F^{\prime} \circ u_{D^{*}}\right]\right)\right|^{p} u \mathrm{~d} x \\
& -\int_{\mathbb{R}^{n}} \nabla\left[F^{\prime} \circ u\right] \cdot \nabla c^{*}\left(\nabla\left[F^{\prime} \circ u_{D^{*}}\right]\right) u \mathrm{~d} x \\
= & \frac{1}{p} \int_{\mathbb{R}^{n}}\left|\nabla\left[F^{\prime} \circ u\right]\right|^{p} u \mathrm{~d} x+\frac{1}{q} \int_{\mathbb{R}^{n}}\left|\left(\nabla\left[F^{\prime} \circ u_{D^{*}}\right]\right)\right|^{p} u \mathrm{~d} x \\
& +\frac{n m}{\gamma} \int_{\mathbb{R}^{n}} u^{\gamma} \mathrm{d} x .
\end{aligned}
$$

Now, we compute the cross term of $\mathbf{I}_{\gamma}^{(\epsilon)}[v(t)]$. We have that

$$
\begin{aligned}
\left.\int_{\mathbb{R}^{n}} \nabla\left[F^{\prime} \circ u\right]\right) \cdot \nabla\left[F^{\prime} \circ u_{D^{*}}\right]( & \left.\epsilon+\left|\nabla\left[F^{\prime} \circ u_{D^{*}}\right]\right|\right)^{p-2} u_{D^{*}} \mathrm{~d} x \\
= & \frac{m}{1-\gamma} \int_{\mathbb{R}^{n}} \nabla\left(u^{\gamma-1}\right) \cdot x|x|^{q-2}\left(\epsilon+|x|^{q-1}\right)^{p-2} u_{D^{*}} \mathrm{~d} x \\
= & -\frac{m}{1-\gamma} \int_{\mathbb{R}^{n}} u^{\gamma-1} \operatorname{div}\left(x|x|^{q-2}\left(\epsilon+|x|^{q-1}\right)^{p-2} u_{D^{*}}\right) \mathrm{d} x \\
= & -\frac{m}{1-\gamma} \int_{\mathbb{R}^{n}} \frac{u^{\gamma}}{w} \operatorname{div}\left(x|x|^{q-2}\left(\epsilon+|x|^{q-1}\right)^{p-2}\right) \mathrm{d} x \\
& +\frac{1}{1-\gamma} \int_{\mathbb{R}^{n}} w^{\gamma-1} u_{D^{*}}|x|^{2(q-1)}\left(\epsilon+|x|^{q-1}\right)^{p-2} \mathrm{~d} x .
\end{aligned}
$$

Since the last term in the above sum is non-negative, then using (4.9) and the fact that $1<p<2$, we can estimate $\mathbf{I}_{\gamma}^{(\epsilon)}[v(t)]$ as

$$
\begin{aligned}
\mathrm{I}_{\gamma}^{(\epsilon)}[v(t)] \leq & \int_{\mathbb{R}^{n}}\left|\nabla\left[F^{\prime} \circ u\right]\right|^{2}\left(\epsilon+\left|\nabla\left[F^{\prime} \circ u_{D^{*}}\right]\right|\right)^{p-2} u_{D^{*}} \mathrm{~d} x \\
& +\int_{\mathbb{R}^{n}}\left|\nabla\left[F^{\prime} \circ u_{D^{*}}\right]\right|^{2}\left(\epsilon+\left|\nabla\left[F^{\prime} \circ u_{D^{*}}\right]\right|\right)^{p-2} u_{D^{*}} \mathrm{~d} x \\
& +\frac{2 m}{1-\gamma} \int_{\mathbb{R}^{n}} \frac{u^{\gamma}}{w} \operatorname{div}\left(x|x|^{q-2}\left(\epsilon+|x|^{q-1}\right)^{p-2}\right) \mathrm{d} x \\
\leq & \int_{\mathbb{R}^{n}}\left|\nabla\left[F^{\prime} \circ u\right]\right|^{2}\left(\epsilon+\left|\nabla\left[F^{\prime} \circ u_{D^{*}}\right]\right|\right)^{p-2} u_{D^{*}} \mathrm{~d} x \\
& +\int_{\mathbb{R}^{n}}\left|\nabla\left[F^{\prime} \circ u_{D^{*}}\right]\right|^{2}\left(\epsilon+\left|\nabla\left[F^{\prime} \circ u_{D^{*}}\right]\right|\right)^{p-2} u_{D^{*}} \mathrm{~d} x \\
& +\frac{2 m(n+2(q-2))}{1-\gamma} \int_{\mathbb{R}^{n}} \frac{u^{\gamma}}{w} \mathrm{~d} x \\
\leq & \int_{\mathbb{R}^{n}}\left|\nabla\left[F^{\prime} \circ u\right]\right|^{p} \Phi_{\epsilon}\left(u, u_{D^{*}}\right)^{2-p} u_{D^{*}} \mathrm{~d} x+\int_{\mathbb{R}^{n}}\left|\nabla\left[F^{\prime} \circ u_{D^{*}}\right]\right|^{p} u_{D^{*}} \mathrm{~d} x \\
& +\frac{2 m(n+2(q-2))}{1-\gamma} \int_{\mathbb{R}^{n}} \frac{u^{\gamma}}{w} \mathrm{~d} x,
\end{aligned}
$$

where

$$
\Phi_{\epsilon}\left(u, u_{D^{*}}\right):=\frac{\left|\nabla\left[F^{\prime} \circ u\right]\right|}{\epsilon+\left|\nabla\left[F^{\prime} \circ u_{D^{*}}\right]\right|} .
$$


Let us check that $\Phi_{\epsilon}\left(u, u_{D^{*}}\right)$ is uniformly bounded. Using Lemma 2.3 we are reduced to work at $|x| \sim \infty$. Actually, we need to show that $\left|\nabla\left(u^{\gamma-1}\right)\right| /\left|\nabla\left(U_{D^{*}}^{\gamma-1}\right)\right|$ is uniformly bounded for $|x|>R$ and $t>t_{0}$. Due to (H1) it is sufficient to prove that $|\nabla u| /\left|\nabla U_{D^{*}}\right|$ is uniformly bounded for $|x|>R$ and $t>t_{0}$. Since $\nabla u=w \nabla U_{D^{*}}+U_{D^{*}} \nabla w$, for $|x|>R$ and $t>t_{0}$ we have

$$
\frac{|\nabla u|}{\left|\nabla U_{D^{*}}\right|} \leq|w|+\frac{|\nabla w|}{\left|\nabla U_{D^{*}}\right|} \nabla U_{D^{*}}
$$

Using (2.3), we have $|\nabla w| \sim O\left(|x|^{-d}\right.$ ) and thus $|\nabla w| /\left|\nabla U_{D^{*}}\right| \nabla U_{D^{*}}$ is bounded for $|x|>R$ and $t>t_{0}$. Therefore $\Phi_{\epsilon}\left(u, u_{D^{*}}\right)$ is uniformly bounded and thus $\Phi_{\epsilon}\left(u, u_{D^{*}}\right) \leq \eta$ for some $\eta$ depending on $\epsilon$ and $t_{0}$. We obtain the estimate

$$
\begin{aligned}
\mathrm{I}_{\gamma}^{(\epsilon)}[v(t)] \leq & \frac{\eta^{2-p}}{W_{0}} \int_{\mathbb{R}^{n}}\left|\nabla\left[F^{\prime} \circ u\right]\right|^{p} u \mathrm{~d} x+\frac{1}{W_{0}} \int_{\mathbb{R}^{n}}\left|\nabla\left[F^{\prime} \circ u_{D^{*}}\right]\right|^{p} u \mathrm{~d} x \\
& +\frac{2 m(n+2(q-2))}{(1-\gamma) W_{0}} \int_{\mathbb{R}^{n}} u^{\gamma} \mathrm{d} x .
\end{aligned}
$$

Combining (4.10) and (4.11), and setting

$$
\delta:=\frac{1}{W_{0}} \max \left(p \eta^{2-p}, q, \frac{2 \gamma(n+2(q-2))}{n(1-\gamma)}\right),
$$

we deduce (4.5).

Proof of Case 2: $2<p<\infty$ (of Claim 2): As above, we have the expression

$$
\mathrm{I}_{\gamma}[v(t)]=\int_{\mathbb{R}^{n}}\left|\nabla\left[F^{\prime} \circ u-F^{\prime} \circ u_{D^{*}}\right]\right|^{2}\left|\nabla\left(F^{\prime} \circ u_{D^{*}}(x)\right)\right|^{p-2} u_{D^{*}} \mathrm{~d} x .
$$

For convenience, we can also rewrite $\mathcal{I}\left(u(t) \mid u_{D^{*}}\right)$ as

$$
\mathcal{I}\left[u(t) \mid u_{D^{*}}\right]=\int_{\mathcal{K}} H\left[u \mid u_{D^{*}}\right]\left|\nabla\left[F^{\prime} \circ u-F^{\prime} \circ u_{D^{*}}\right]\right|^{2} u \mathrm{~d} y
$$

where

$$
H\left[u(t) \mid u_{D^{*}}\right]=\frac{\nabla\left(F^{\prime} \circ u-F^{\prime} \circ u_{D^{*}}\right) \cdot\left[\nabla c^{*}\left[\nabla\left(F^{\prime} \circ u\right)\right]-\nabla c^{*}\left[\nabla\left(F^{\prime} \circ u_{D^{*}}\right)\right]\right]}{\left|\nabla\left(F^{\prime} \circ u-F^{\prime} \circ u_{D^{*}}\right)\right|^{2}}
$$

and $\mathcal{K}:=\left\{x \in \mathbb{R}^{n}\right.$ such that $\left.\left|\nabla\left[F^{\prime} \circ u-F^{\prime} \circ u_{D^{*}}\right]\right| \neq 0\right\}$. Let us show that there exist a constant $\delta>0$, such that for all $t>t_{0}$,

$$
H\left[u(t) \mid u_{D^{*}}\right] \geq \delta\left|\nabla\left(F^{\prime} \circ u_{D^{*}}\right)\right|^{p-2} .
$$

Let us remark, if $p=2$, then $\delta=1$, and equality holds in (4.12).

For simplicity, set $a(t)=\nabla\left(F^{\prime} \circ u(t)\right)$ and $a_{D^{*}}=\nabla\left(F^{\prime} \circ u_{D^{*}}\right)$. It is clear that (4.12) holds in the set where $a_{D^{*}}=0$. Therefore, let us restrict to the set where $a_{D^{*}} \neq 0$ without loss of generality. Let us denote $b(t)=a(t) /\left|a_{D^{*}}\right|$ and $b_{D^{*}}=a_{D^{*}} /\left|a_{D^{*}}\right|$. It is straightforward to check that

$$
\frac{H\left[u(t) \mid u_{D^{*}}\right]}{\left|\nabla\left(F^{\prime} \circ u_{D^{*}}\right)\right|^{p-2}}=\frac{\left(b(t)-b_{D^{*}}\right) \cdot\left(|b(t)|^{p-2} b(t)-b_{D^{*}}\right)}{\left|b(t)-b_{D^{*}}\right|^{2}} .
$$


Let $\theta$ denote the angle between $b(t)$ and $b_{D^{*}}$. We have that

$$
\left|b-b_{D^{*}}\right|^{2}=|b|^{2}+\left|b_{D^{*}}\right|^{2}-2 b \cos \theta=1+|b|^{2}-2 b \cos \theta
$$

and

$$
\left.\left(b-b_{D^{*}}\right) \cdot\left(|b|^{p-2} b-b_{D^{*}}\right)\right)=|b|^{p}-|b| \cos \theta-|b|^{p-1} \cos \theta+\left|b_{D^{*}}\right|^{2}=1+|b|^{p}-\left(|b|+|b|^{p-1}\right) \cos \theta
$$

so that (4.13) reads as:

$$
\frac{H\left[u(t) \mid u_{D^{*}}\right]}{\left|\nabla\left(F^{\prime} \circ u_{D^{*}}\right)\right|^{p-2}}=\frac{1+r(t)^{p}-\left(r(t)+r(t)^{p-1}\right) x(t)}{1+r(t)^{2}-2 r(t) x(t)}
$$

where $r(t)=|b(t)| \geq 0$ and $x(t)=\cos \theta \in[-1,1]$, with $r(t) \rightarrow 1$ as $t \rightarrow \infty$. Estimate (4.12) is reduced to show that

$$
f_{p}(r, x):=\frac{1+r^{p}-\left(r+r^{p-1}\right) x}{1+r^{2}-2 r x} \geq \delta
$$

for all $x \in[-1,1]$ and for all $r \geq 0$. For that, let us define the function

$$
F_{p}(r, x):=1+r^{p}-\left(r+r^{p-1}\right) x-\delta\left(1+r^{2}-2 r x\right),
$$

which is easily checked to be decreasing in $x$ for $r>0$, whenever $\delta \leq \frac{1}{2}$. Therefore, we have $F_{p}(r, x)>F_{p}(r, 1)$ and thus, to show (4.14) for $r>0$ and $-1 \leq x<1$ is reduced to show that $F_{p}(r, 1) \geq 0$, whenever $\delta \leq \frac{1}{2}$. Since $F_{p}(1,1)=0$, this is equivalent to show that $f_{p}(r, 1) \geq \delta$ for $0<r<1$ and $r>1$. The last assertion comes from the fact that when $p>2$,

$$
f_{p}(r, 1)=\frac{\left(r^{p}-r\right)-\left(r^{p-1}-1\right)}{(r-1)^{2}}=\frac{(r-1)\left(r^{p-1}-1\right)}{(r-1)^{2}}=\frac{r^{p-1}-1}{r-1}
$$

is bounded below by 1 , since in $0<r<1$, we have $r^{p-1}-1<r-1<0$; in $r>1$, we have $r^{p-1}-1>r-1>0$; and $\lim _{r \rightarrow 1} f_{p}(r, 1)=p-1>1$. Therefore,

$$
f_{p}(r, 1) \geq 1>\frac{1}{2} \geq \delta \text {. }
$$

Proof of the main theorem, Theorem 1.1: Given $1<p<2$ and $\epsilon>0$, set $v=u-u_{D^{*}}$. From Proposition 4.2 and the strong weighted Poincaré inequality (3.17), we have that

$$
\mathrm{E}[v(t)] \leq \frac{\kappa_{1} \tilde{\beta}_{\epsilon}}{2-\kappa_{2} \tilde{\beta}_{\epsilon}} \mathcal{I}\left[u(t) \mid u_{D^{*}}\right],
$$

where $\kappa_{2}$ can be chosen arbitrary small provided that $t>t_{0}$ is large enough. This together with proposition 4.1 yields the Hardy-Poincaré type inequality:

$$
\mathcal{E}\left[u(t) \mid u_{D^{*}}\right] \leq \frac{1}{\lambda} \mathcal{I}\left[u(t) \mid u_{D^{*}}\right],
$$

where $\lambda:=\left(2-\kappa_{2} \tilde{\beta}_{\epsilon}\right) / C_{2} \kappa_{1} \tilde{\beta}_{\epsilon}>0$. We combine (4.15), $\left.\lim _{t \rightarrow \infty} \mathcal{E}[u(t)) \mid u_{D_{*}}\right]=0$ (see Proposition 2.6) and the entropy dissipation equation

$$
\frac{\mathrm{d}}{\mathrm{d} t} \mathcal{E}\left[u(t) \mid u_{D_{*}}\right]=-\mathcal{I}\left[u(t) \mid u_{D_{*}}\right]
$$


to obtain the exponential decay of the relative entropy, $\mathcal{E}\left[u(t) \mid u_{D^{*}}\right] \leq e^{-\lambda t} \mathcal{E}\left[u_{0} \mid u_{D^{*}}\right]$. The $L^{1}$ decay (1.13) follows from the Csiszàr-Kullback type inequality (see for e.g., [3]),

$$
\left\|u(t)-u_{D^{*}}\right\|_{L^{1}\left(\mathbb{R}^{n}\right)}^{2} \leq M(n, n, p) \mathcal{E}\left[u(t) \mid u_{D^{*}}\right], \quad M(m, n, p)>0,
$$

and (1.14) is a direct consequence of the rescaling (1.7)-(1.8). The case $p>2$ follows analogously without need of using the regularised entropy dissipation.

Remark 4.3 The rate of convergence $\lambda$ in Theorem 1.1 can be explicitly reconstructed in the above computation for a given choice of $\epsilon$ for $p<2$, but $t_{0}$ will depend on the choice of $\epsilon$. For $p>2$, we can even give an explicit range for the constant $\lambda$.

Acknowledgements: MA is partially supported by a grant from NSERC (Canada). JAC acknowledge partial support from the project MTM2008-06349-C03-03 from DGI-MCI (Spain). We also thank WPI-Vienna where this research was started in the framework of the program "Optimal transportation structures, gradient flows and entropy methods for Applied PDEs". $\mathrm{AB}$ and JAC acknowledges IPAM-UCLA where part of this work was done.

This paper is under the Creative Commons licence Attribution-NonCommercialShareAlike 2.5.

\section{References}

[1] M. Agueh, Asymptotic behavior for doubly degenerate parabolic equations, C. R. Math. Acad. Sci. Paris, Ser. I 337 (2003) 331-336.

[2] M. Agueh, Existence of solutions to degenerate parabolic equations via the Monge-Kantorovich theory, Adv. Differential Equations., Vol.10, 3 (2005), 309-360.

[3] M. Agueh, Rates of decay to equilibria for p-Laplacian type equations, Nonlinear Analysis, 68 (2008), 1909-1927.

[4] G. I. Barenblatt, On self-similar motions of compressible fluids in porous media, Prikl. Math. 16 (1952) 679-698 (in Russian).

[5] A. Blanchet, M. Bonforte, J. Dolbeault, G. Grillo, J. L. Vázquez, Hardy-Poincaré inequalities and applications to nonlinear diffusions, C. R. math. Acad. Sci. Paris. 344 (2007) 431-436.

[6] A. Blanchet, M. Bonforte, J. Dolbeault, G. Grillo, J. L. Vázquez, Asymptotic of the fast diffusion equation via entropy estimates, to appear in Arch. Rational. Mech. Anal.

[7] M. Bonforte, J. L. Vázquez, Global positivity estimates and Harnack inequalities for the fast diusion equation, J. Funct. Anal. 240 (2006) 399-428.

[8] N. Calvo, J.I. Díaz, J. Durany, E. Schiavi, C. Vázquez, On a doubly nonlinear parabolic obstacle problem modeling ice sheet dynamics, SIAM J. App. Math. 63 (2002), 683-707.

[9] J. A. Carrillo, G. Toscani, Asymptotic $L^{1}$-decay of solutions of the porous medium equation to selfsimilarity, Indiana Univ. math. J. 49 (2000) 113-141.

[10] J. A. Carrillo, C. Lederman, P. A. Markowich, G. Toscani, Poincaré inequalities for linearization of very fast diffusion equations, Nonlinearity 15 (2002) 565-580. 
[11] J. A. Carrillo, J. L. Vázquez, Fine asymptotics for fast diffusion equations, Comm. Partial Differential Equations 28 (2003) 1023-1056.

[12] J. Denzler, R. J. McCann, Fast diffusion to self-similarity: complete spectrum, long time asymptotics and numerology, Arch. Rational Mech. Anal. 179 (2006) 217-263.

[13] E. DiBenedetto, Degenerate parabolic equations, Springer-Verlag, Berlin Heidelberg New York, 1993.

[14] E. DiBenedetto, M. A. Herrero, Nonnegative solutions of the evolution p-Laplacian equation. Initial traces and Cauchy problem when $1<p<2$, Arch. Rational Mech. Anal. 111 (1990) 225-290.

[15] E. DiBenedetto, M. A. Herrero, On the Cauchy problem and initial traces for a degenerate parabolic equation, Trans. Amer. Math. Soc. 314 (1989), no. 1, 187-224.

[16] J. Dolbeault, M. Del Pino, Best constants for Gagliardo-Nirenberg inequalities and applications to nonlinear diffusions, J. Math. Pures Appl. 81 (2002) 847-875.

[17] M. Del Pino, J. Dolbeault, Nonlinear diffusion and optimal constants in Sobolev type inequalities: asymptotic behaviour of equations involving p-Laplacian, C. R. Math. Acad. Sci. Paris, Ser. I 334 (2002) 365-370 .

[18] M. Del Pino, J. Dolbeault, Asymptotic behaviour of nonlinear diffusions, Math. Res. Lett. 10 (4) (2003) 551-557.

[19] A. Friedmann, S. Kamin, The asymptotic behaviour of gas in a n-dimensional porous media, Trans. Amer. Math. Soc. 262 (2) (1980) 551-563.

[20] K. H. Hutter, Mathematical foundation of ice sheet and ice shelf dynamics: A physicist's view, Free boundary problems: theory and applications, 192-20, Chapman \& Hall/CRC Res. Notes math., 409, Boca Raton, FL, 1999.

[21] Y. J. Kim, R. J. McCann, Potential theory and optimal convergence rates in fast nonlinear diffusion, J. Math. Pures Appl. 86 (2006) 42-67.

[22] S. Kamin, J. L. Vázquez, Fundamental solutions and asymptotic behaviour for the p-Laplacian equation, Rev. Mat. Iberoamericana 4 (1988) 339-354.

[23] O. A. Ladyzhenskaya, The mathematical theory of viscous incompressible flow, Gordon and Breach, 1969.

[24] O. A. Ladyženskaja, V. A. Solonnikov, N. N. Uralceva, Linear and quasilinear equations of parabolic type, American Mathematical Society, 1967.

[25] L. S. Leibenzon, General problem of the movement of a compressible fluid in a porous medium, Ivz. Acad. nauk. SSSR, Geography and Geophysics 9 (1945), 7-10 (in Russian).

[26] J. Li, Cauchy problem and initial trace for a doubly degenerate parabolic equation with strongly nonlinear sources, J. Math. Anal. Appl. 264 (2001) 4967.

[27] G. M. Lieberman, Second order parabolic differential equations, World Scientific Publishing Co. Inc., 1996.

[28] R. J. McCann, A convexity principle for interacting gases, Adv. math. 128 (1997) 153-179.

[29] R. J. McCann, D. Slepčev, Second-order asymptotics for the fast-diffusion equation, Int. Math. Res. Not. (2006) Art. ID 24947, 22. 
[30] L. Nirenberg, On elliptic partial differential equations, Ann. Scuola Norm. Sup. Pisa 13 (1959) 115162.

[31] F. Otto, The geometry of dissipative evolution equations: the porous medium equation, Comm. Partial. Differential Equations 269 (2001) 101-174.

[32] A. Persson, Bounds for the discrete part of the spectral of a semi-bounded Schrödinger operator, Math. Scand. 8 (1960) 143-153.

[33] J. L. Vázquez, Smoothing and decay estimates for nonlinear diffusion equations, Oxford lecture series in mathematics and its applications, 33, Oxford Univ. Press, 2006. 